\title{
Self-acceleration of a charge intersecting a boundary surface in a waveguide
}

\author{
Tatiana Yu. Alekhina* and Andrey V. Tyukhtin ${ }^{\dagger}$ \\ Physical Faculty of St. Petersburg State University, St. Petersburg 198504, Russia
}

(Received 23 January 2013; published 12 August 2013)

\begin{abstract}
We analyze the interaction between a charge and its electromagnetic field in the case of the charge moving in a waveguide and intersecting a boundary between two nondispersive media. The total work done by the field on the charge is calculated both analytically and numerically. It is shown that the work is positive under certain conditions, that is the force actuating the charge is accelerating. A physical interpretation of this phenomenon from the point of view of energy conservation is given. The kinetic energy gain per unit length and for all time of motion is analyzed as well.
\end{abstract}

DOI: 10.1103/PhysRevSTAB.16.081301

PACS numbers: 41.75.Lx, 41.60.Dk

\section{PHYSICAL CONSIDERATION}

All particle accelerators use "external" electromagnetic fields to increase the energy of the particles. The self-fields of accelerated particles play negative roles in the acceleration process; they result in the deceleration and instability of particle bunches. However, an interesting question is the following: can we obtain the inverse effect in which the particle bunch is accelerated as a result of the interaction between the bunch and its self-field? It is clear that such a phenomenon is not possible in any regular structures with characteristics that do not depend on the coordinate along the bunch trajectory. However, this phenomenon may be probable in irregular structures.

For example, vacuum perfectly conducting pipes with an abrupt change in their cross sections are such structures. A "step-out" case, when a particle moves into a pipe of a bigger radius, and a "step-in" case, when a particle enters into a narrowing pipe, are possible. As it was shown [1-3], a bunch loses energy in the step-out case, but would regain some amount of energy in the step-in case. The effect of acceleration or deceleration of a charge in such a waveguide is due to the asymmetric discontinuous boundary conditions.

Analogues phenomena take place when the charge intersects the boundary between two media [4]. A balance of energy for such a problem was considered in [4] where the acceleration/deceleration phenomenon was explained in terms of macroscopic mass renormalization. We will analyze here analogous effects in a waveguide with irregular filling.

Let us consider a circular waveguide and a point charge $q$ moving along the waveguide axis $z$ with velocity $\vec{V}=$ $c \beta \vec{e}_{z}$ (where $c$ is the speed of light in vacuum). We assume that the charge velocity is essentially constant but that the

\footnotetext{
*tanya@niirf.spbu.ru

†tyukhtin@bk.ru
}

Published by the American Physical Society under the terms of the Creative Commons Attribution 3.0 License. Further distribution of this work must maintain attribution to the author(s) and the published article's title, journal citation, and DOI. charge kinetic energy can vary. This assumption is exactly fulfilled only for a particle with infinite mass. However, this assumption is approximately true for many situations, e.g., a large series of problems concerning Cherenkov and transition radiation in which the approximation $\vec{V} \approx$ const holds true. The waveguide under consideration is filled with a dielectric material with permittivity $\varepsilon_{1}$ for $z<0$ and $\varepsilon_{2}$ for $z>0$ (Fig. 1). For simplicity, the permeability is assumed to be 1 everywhere.

Note that a vacuum channel is usually required for the unimpeded motion of a particle bunch. However, we assume that the channel radius is much smaller than the waveguide radius $a$. Under this condition, the series of waveguide modes has a typical field variation scale that is much larger than the channel radius. The influence of the channel on these modes is negligible [5], and we can consider a waveguide without a channel.

It is assumed that Cherenkov radiation is not generated anywhere, i.e., the charge velocity is less than the phase velocities of the waves in the media, $V<c / \sqrt{\varepsilon_{1,2}}$. We first consider large $z$ distances $(z \rightarrow \pm \infty)$, where the transition radiation field is separate from the charge. In these areas, the self-field of the charge is the same as the field in the regular waveguide that has a homogeneous filling (this field is the so-called "forced" field [4]). Using cylindrical coordinates, $r, \phi, z$, the nonzero components of the forced field can be written in the following mode expansion form [5-7]:

$$
E_{z, r}=\sum_{n=1}^{\infty} E_{n z, r}, \quad H_{\phi}=\sum_{n=1}^{\infty} H_{n \phi}
$$

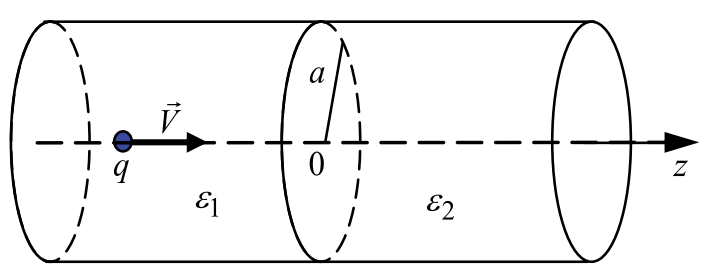

FIG. 1. Geometry of the problem. 


$$
\begin{aligned}
\left\{\begin{array}{c}
E_{n z}^{q} \\
E_{n r}^{q} \\
H_{n \phi}^{q}
\end{array}\right\}= & \frac{2 q}{a^{2}}\left\{\begin{array}{c}
-\varepsilon^{-1} J_{0}\left(\chi_{n} r / a\right) \\
\varepsilon^{-1}\left(1-\beta^{2} \varepsilon\right)^{-1 / 2} J_{1}\left(\chi_{n} r / a\right) \\
\beta\left(1-\beta^{2} \varepsilon\right)^{-1 / 2} J_{1}\left(\chi_{n} r / a\right)
\end{array}\right\} J_{1}^{-2}\left(\chi_{n}\right) \\
& \times \exp \left[-\frac{\omega_{n}|\zeta|}{c \sqrt{1-\beta^{2} \varepsilon}}\right]
\end{aligned}
$$

where $J_{0,1}(x)$ are Bessel functions, $\chi_{n}$ is the $n$th zero of the Bessel function $J_{0}(x), \omega_{n}=\chi_{n} c / a, \zeta=z-V t, \varepsilon=\varepsilon_{1}$ for $z \rightarrow-\infty$, and $\varepsilon=\varepsilon_{2}$ for $z \rightarrow+\infty$.

The energy of a field in a waveguide is the sum of the energies of the modes $W^{(1,2)}=\sum_{n} W_{n}^{(1,2)}$, where indices 1 and 2 correspond to $z \rightarrow-\infty$ and $z \rightarrow \infty$, respectively. Calculating the mode energy gives

$$
\begin{aligned}
W_{n}^{(1,2)} & =\frac{1}{8 \pi} \int_{0}^{2 \pi} d \phi \int_{0}^{a} r d r \int_{-\infty}^{\infty} d z\left(\varepsilon E_{n r}^{2}+\varepsilon E_{n z}^{2}+\mu H_{n \phi}^{2}\right) \\
& =\frac{q^{2}}{\varepsilon_{1,2} a \sqrt{1-\beta^{2} \varepsilon_{1,2}} \chi_{n}} J_{1}^{2}\left(\chi_{n}\right)
\end{aligned}
$$

Here, we use the tabulated integrals of the functions of type $x J_{0,1}^{2}(x)$ [8]. The differences in the mode energies of the forced field are

$$
\begin{aligned}
\Delta W_{n} & =W_{n}^{(2)}-W_{n}^{(1)} \\
& =\frac{q^{2}}{a \chi_{n} J_{1}^{2}\left(\chi_{n}\right)}\left(\frac{1}{\varepsilon_{2} \sqrt{1-\beta^{2} \varepsilon_{2}}}-\frac{1}{\varepsilon_{1} \sqrt{1-\beta^{2} \varepsilon_{1}}}\right) .
\end{aligned}
$$

One can see that $\Delta W_{n}<0$ under the conditions

$$
\begin{aligned}
& \beta^{2}<\frac{\varepsilon_{2}^{2}-\varepsilon_{1}^{2}}{\varepsilon_{2}^{3}-\varepsilon_{1}^{3}} \quad \text { for } \varepsilon_{2}>\varepsilon_{1}, \\
& \beta^{2}>\frac{\varepsilon_{1}^{2}-\varepsilon_{2}^{2}}{\varepsilon_{1}^{3}-\varepsilon_{2}^{3}} \quad \text { for } \varepsilon_{1}>\varepsilon_{2} .
\end{aligned}
$$

Thus, under one of these conditions, the self-field energy for $z \rightarrow+\infty$ is less than the energy for $z \rightarrow-\infty$.

It appears likely that the energy $\left|\Delta W_{n}\right|$ is only spent on transition radiation. However, in the nonrelativistic case $(\beta \ll 1)$, the transition radiation energy is proportional to $\beta^{2}$, i.e., it is negligible for very slow motion (an "adiabatic" regime). At the same time, $\Delta W_{n}$ is finite,

$$
\Delta W_{n} \approx \frac{q^{2}}{a \chi_{n} J_{1}^{2}\left(\chi_{n}\right)} \frac{\varepsilon_{1}-\varepsilon_{2}}{\varepsilon_{1} \varepsilon_{2}},
$$

and is negative if $\varepsilon_{2}>\varepsilon_{1}$.

However, in accordance with the energy conservation law, the kinetic energy of the charge must be taken into account. This law can be written in the following form:

$$
W^{(1)}+K^{(1)}=W^{(2)}+K^{(2)}+\Sigma,
$$

where $K^{(1,2)}$ are the kinetic energies of the charge at $z \rightarrow \mp \infty$, and $\Sigma$ is the transition radiation energy. In the case of a slow velocity $(\beta \ll 1)$, we have

$$
K^{(2)}-K^{(1)} \approx W^{(1)}-W^{(2)}=-\sum_{n} \Delta W_{n} .
$$

Thus, for $\beta \ll 1$ under the first from conditions (5), the kinetic energy gain (KEG) $\Delta K=K^{(2)}-K^{(1)}$ is positive, i.e., the charge is accelerated. We will further specify the conditions for this phenomenon.

\section{CALCULATION OF THE KINETIC ENERGY GAIN (KEG)}

The KEG $\Delta K=K^{(2)}-K^{(1)}$ can be calculated using law (7). However, it is simpler to use the work done by the field on the charge $\Delta K=A=\sum A_{n}$, where $A_{n}$ is the work done by the field of the $n$ mode:

$$
A_{n}=\int_{-\infty}^{+\infty} F_{n z} d z=\left.q V \int_{-\infty}^{+\infty} E_{n z}\right|_{r=0, z=V t} d t .
$$

This approach is more obvious because we directly consider the strength affecting the charge $F_{n z}=\left.q E_{n z}\right|_{r=0, z=V t}$.

The total field of the mode contains both the forced field (2) and the "free" one (the latter is related to the boundary influence). The longitudinal component of the free field can be written in the form [6,7]

$$
\begin{aligned}
E_{z n 1,2}^{b}= & \frac{2 i q \beta \omega_{n}^{2} J_{0}\left(\chi_{n} r / a\right)}{\pi a^{2} \varepsilon_{1,2} J_{1}^{2}\left(\chi_{n}\right)} \\
& \times \int_{-\infty}^{+\infty} d \omega B_{n 1,2} \exp \left[i\left(k_{z 1,2}|z|-\omega t\right)\right],
\end{aligned}
$$

where

$$
\begin{aligned}
& B_{n 1,2}=\frac{\left(\varepsilon_{1,2}-\varepsilon_{2,1}\right)\left[\omega\left( \pm \varepsilon_{1,2} \beta^{2} \mp 1\right)-\beta \sqrt{\omega^{2} \varepsilon_{2,1}-\omega_{n}^{2}}\right]}{g(\omega)\left[\omega^{2}\left(1-\varepsilon_{1,2} \beta^{2}\right)+\beta^{2} \omega_{n}^{2}\right]\left(\omega \pm \beta \sqrt{\omega^{2} \varepsilon_{2,1}-\omega_{n}^{2}}\right)} \\
& k_{z 1,2}=\frac{1}{c} \sqrt{\omega^{2} \varepsilon_{1,2}-\omega_{n}^{2}}, \quad \omega_{n}=\chi_{n} c / a, \\
& g(\omega)=\varepsilon_{1} \sqrt{\omega^{2} \varepsilon_{2}-\omega_{n}^{2}}+\varepsilon_{2} \sqrt{\omega^{2} \varepsilon_{1}-\omega_{n}^{2}} .
\end{aligned}
$$

The indices 1 and 2 correspond to the areas $z<0$ and $z>0$, respectively.

Because Cherenkov radiation is not generated anywhere, the forced field $\vec{E}^{q}$ is a "quasi-Coulomb" one, and this field does not take any energy from the charge. Therefore, only the free field $\vec{E}^{b}$ affects the charge. Using (9) and (10), we obtain

$$
\begin{aligned}
A_{n}= & A_{n 1}+A_{n 2}=q V \int_{-\infty}^{0} E_{z 1}^{b} d t+q V \int_{0}^{+\infty} E_{z 2}^{b} d t \\
= & \frac{2 q^{2} c^{3} \beta^{2} \chi_{n}^{2}}{\pi a^{4} J_{1}^{2}\left(\chi_{n}\right)}\left(-\frac{1}{\varepsilon_{1}} \int_{-\infty}^{+\infty} \frac{B_{n 1}}{\omega+V k_{z 1}} d \omega\right. \\
& \left.+\frac{1}{\varepsilon_{2}} \int_{-\infty}^{+\infty} \frac{B_{n 2}}{\omega-V k_{z 2}} d \omega\right) .
\end{aligned}
$$

The integration with respect to $t$ is performed using the assumption that $k_{z 1,2}$ have very small positive imaginary 


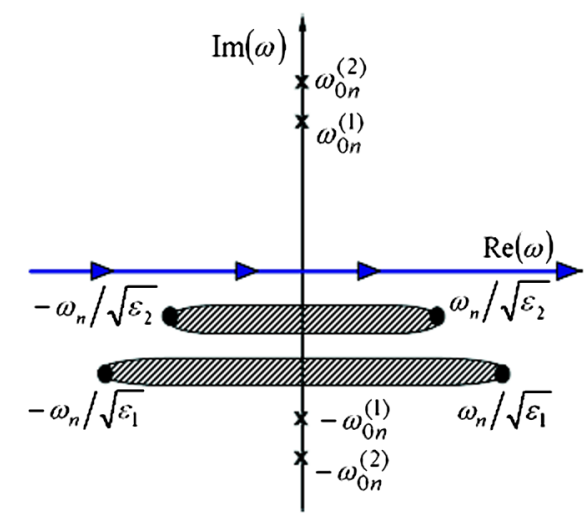

FIG. 2. Poles (crosses), branch points (circlets), branch cuts, and the integration path in a complex plane of $\omega$ for integrals in (11).

parts (i.e., we take into account the very small losses in the media).

It can be shown that the integration path in (11) goes along the upper edge of the branch cuts in a complex plane of $\omega$ (Fig. 2). The integrands in (11) behave as $\omega^{-4}$ at $|\omega| \rightarrow \infty$. Therefore, we can close the integration contour in the upper half-plane $(\operatorname{Im} \omega>0)$, where the first integrand has the simple pole $\omega_{0 n}^{(1)}=i \beta \omega_{n} / \sqrt{1-\beta^{2} \varepsilon_{1}}$ and the second integrand has the same simple pole $\omega_{0 n}^{(1)}$ and the 2-d order pole $\omega_{0 n}^{(2)}=i \beta \omega_{n} / \sqrt{1-\beta^{2} \varepsilon_{2}}$ (Fig. 2). Using the residue theorem, we obtain the following expressions:

$$
\begin{aligned}
A_{n 1}= & \frac{q^{2}\left(\varepsilon_{2}-\varepsilon_{1}\right)}{a \varepsilon_{1} \chi_{n} J_{1}^{2}\left(\chi_{n}\right)} \frac{\sqrt{1-\beta^{2} \varepsilon_{1}}\left(1-\beta^{2} \varepsilon_{1}+S_{+}\right)}{\left(1+S_{+}\right)\left(\varepsilon_{2}+\varepsilon_{1} S_{+}\right)} \\
A_{n 2}= & -\frac{q^{2}}{a \chi_{n} J_{1}^{2}\left(\chi_{n}\right)}\left\{\frac{4 \sqrt{1-\beta^{2} \varepsilon_{1}}}{\left(1-S_{+}\right)\left(\varepsilon_{2}+\varepsilon_{1} S_{+}\right)}+\frac{\sqrt{1-\beta^{2} \varepsilon_{2}}}{\varepsilon_{2}}\right. \\
& \left.+\frac{2\left[1-\beta^{2} \varepsilon_{2}+\left(1-\beta^{2} \varepsilon_{1}\right) S_{-}\right]}{\left(\varepsilon_{1}-\varepsilon_{2}\right) \sqrt{1-\beta^{2} \varepsilon_{2}}\left(1-\beta^{2} \varepsilon_{2}-S_{-}\right)}\right\}
\end{aligned}
$$

where $S_{ \pm}=\sqrt{1 \pm \beta^{2}\left(\varepsilon_{2}-\varepsilon_{1}\right)}$.

The exact formula (12) leads to the following expression in the nonrelativistic case $(\beta \ll 1)$ :

$$
A_{n} \approx \frac{q^{2}\left(\varepsilon_{2}-\varepsilon_{1}\right)}{a \chi_{n} J_{1}^{2}\left(\chi_{n}\right)}\left[\frac{1}{\varepsilon_{1} \varepsilon_{2}}+2 \frac{\left(\varepsilon_{1}-\varepsilon_{2}\right)}{\left(\varepsilon_{1}+\varepsilon_{2}\right)^{2}} \beta^{2}\right] .
$$

Thus, this value is positive when $\varepsilon_{2}>\varepsilon_{1}$, that is the charge is accelerated during its motion through the boundary. If we neglect the second-order term $\sim \beta^{2}$ in (13), we obtain $A_{n} \approx-\Delta W_{n}(6)$. This result is explained by the fact that the transition radiation is negligible in this approximation.

Another simple approximation can be obtained in the case of the charge velocity being close to the phase velocity in the dielectric. If $\varepsilon_{1}<\varepsilon_{2}$ and $1-\beta^{2} \varepsilon_{2} \ll 1$, then we have

$$
A_{n} \approx A_{n 2} \approx-\frac{2 q^{2}}{a \chi_{n} J_{1}^{2}\left(\chi_{n}\right) \varepsilon_{2} \sqrt{1-\beta^{2} \varepsilon_{2}}} .
$$

As seen, this value is negative and tends to $-\infty$ at $\beta^{2} \varepsilon_{2} \rightarrow 1$, i.e., the charge is decelerated for velocities closed to the Cherenkov threshold $\beta_{C 2}=1 / \sqrt{\varepsilon_{2}}$. Thus, if $\varepsilon_{1}<\varepsilon_{2}$, then the charge acceleration effect occurs when $\beta<\beta_{0}$, where the threshold value $\beta_{0}$ is less than $\beta_{C 2}$.

If $\varepsilon_{1}>\varepsilon_{2}$ and $\beta=1 / \sqrt{\varepsilon_{1}}$, then

$$
A_{n} \approx \frac{-q^{2} \sqrt{1-\tilde{\varepsilon}}}{a \varepsilon_{1} \chi_{n} J_{1}^{2}\left(\chi_{n}\right)}\left(\frac{1}{\tilde{\varepsilon}}+\frac{2}{(1-\tilde{\varepsilon})(1-\tilde{\varepsilon}-\sqrt{2-\tilde{\varepsilon}})}\right),
$$

where $\tilde{\varepsilon}=\varepsilon_{2} / \varepsilon_{1}$. This value is positive for $\tilde{\varepsilon} \geq 0.216$. Thus, if $\varepsilon_{1}>\varepsilon_{2}$, the effect of the charge acceleration takes place at $\beta>\beta_{1}$, where the threshold value $\beta_{1}$ is less than the Cherenkov threshold $\beta_{C 1}=1 / \sqrt{\varepsilon_{1}}$.

We note that in the case of $\beta>\beta_{C 1}$ or $\beta>\beta_{C 2}$, the total radiation losses are infinite because Cherenkov radiation is generated from the infinite pass of the charge in the dielectric. In the case considered here, when Cherenkov radiation is not generated $\left(\beta<\beta_{C 1,2}\right)$, this effect is absent because the transition radiation formation distance is finite [4].

\section{NUMERICAL RESULTS AND DISCUSSION}

The KEG for the 1st mode $K_{1}^{(2)}-K_{1}^{(1)}=A_{1}$ is shown in Fig. 3. We note that the dependences of $A_{n}$ on $\beta$ and permittivity $\varepsilon_{2}$ or $\varepsilon_{1}$ have the same characteristics for all of the modes; in accordance with (12), the differences consist of the multipliers $\chi_{n}^{-1} J_{1}^{-2}\left(\chi_{n}\right)$ only.

For the case when $\varepsilon_{1}<\varepsilon_{2}, A_{n}$ is positive for a large range of velocities $\beta<\beta_{0}$ (Fig. 3, left). The velocity limit of this effect $\beta_{0}$ does not depend on the mode number and decreases with increasing permittivity (Fig. 4, top). The magnitude of the field work at $\beta \rightarrow 0$ has a maximum, and it increases with increase in $\varepsilon_{2}$ (Fig. 5, top).

For the case when $\varepsilon_{1}>\varepsilon_{2}, A_{n}$ is negative at $\beta<\beta_{1}$, and positive at $\beta_{1}<\beta<\beta_{C 1}$ (Fig. 3, right). The threshold velocity $\beta_{1}$ decreases with increase in $\varepsilon_{1}$ (Fig. 4 , bottom). The magnitude of the field work has a maximum at the Cherenkov threshold $\beta_{C 1}$, and it decreases with increase in permittivity $\varepsilon_{1}$ (Fig. 5, bottom). This maximum is positive for $\varepsilon_{2} \geq 0.216 \varepsilon_{1}$ (i.e. $\varepsilon_{1} \leq 4.624$ if $\varepsilon_{2}=1$ ).

It is interesting to consider the two following energetic characteristics: the KEG (the field work) per unit length of the charge pass

$$
d A_{n} / d z=\left.q E_{n z}^{b}\right|_{r=0, z=V t}
$$

and the KEG (the field work) during the time interval $(-\infty, t)$

$$
\Delta A_{n}(t)=\left.q V \int_{-\infty}^{t} E_{n z}^{b}\right|_{r=0, z=V t^{\prime}} d t^{\prime} .
$$

The typical dependences of these values on time for the case when the charge is flying from vacuum into dielectric 

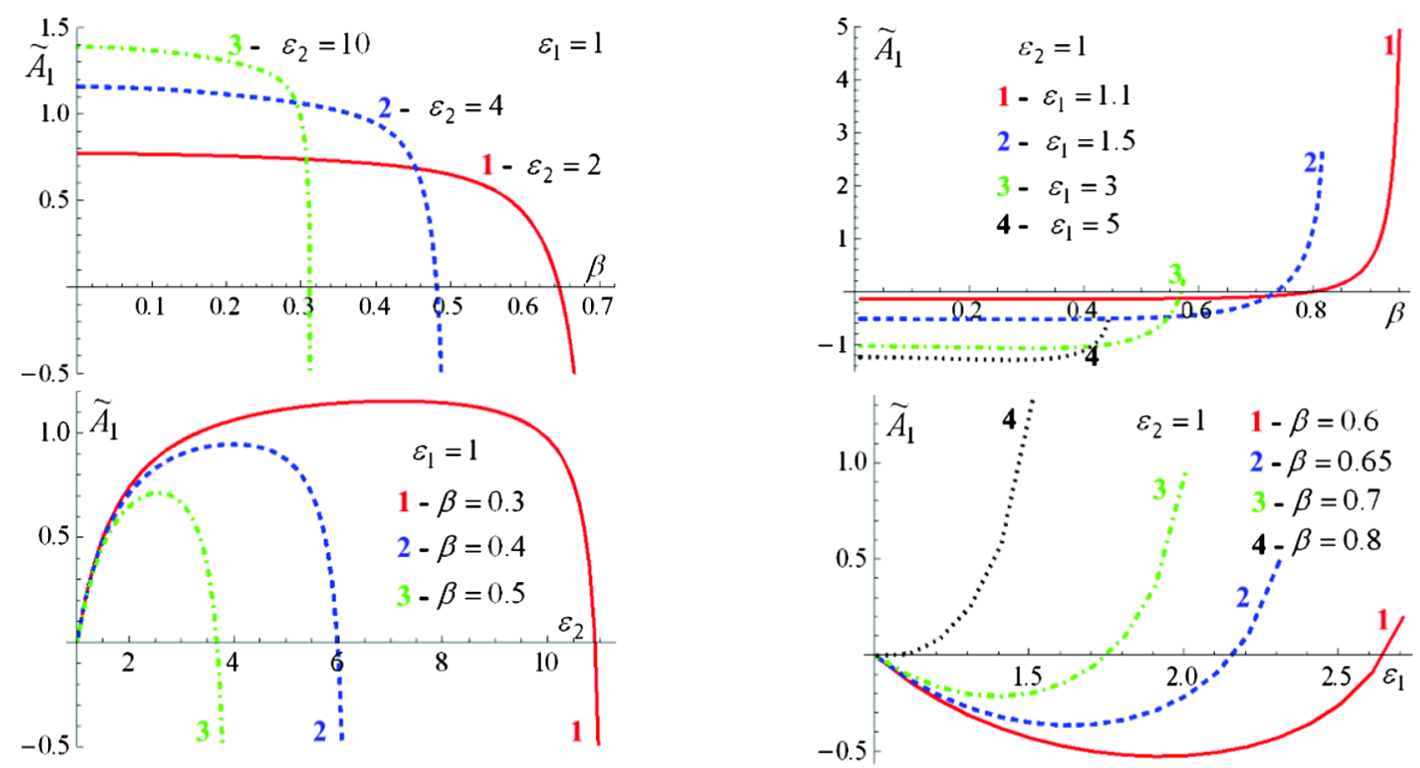

FIG. 3. Dependence of the normalized KEG $\tilde{A}_{1}=A_{1} a / q^{2}$ on $\beta$ for different values of permittivity $\varepsilon_{2}$ or $\varepsilon_{1}$ (top) and on $\varepsilon_{2}$ or $\varepsilon_{1}$ for different values of $\beta$ (bottom); the case of $\varepsilon_{2}>\varepsilon_{1}=1$ is presented on the left, and the case of $\varepsilon_{1}>\varepsilon_{2}=1$ is shown on the right.

are shown in Fig. 6. It can be seen that the KEG per unit length is positive when $t<0$ and has a maximum at $t=-0$. After the particle flies into the medium, $d A_{n} / d z$ remains positive for some time and then oscillates near the zero level (this result is explained by the interaction between the charge and the transition radiation). This phenomenon occurs when the velocity $\beta<\beta_{C 2}$. However, the area with positive values of $d A_{n} / d z$ is more essential for small velocities [Figs. 6(a) and 6(b)]
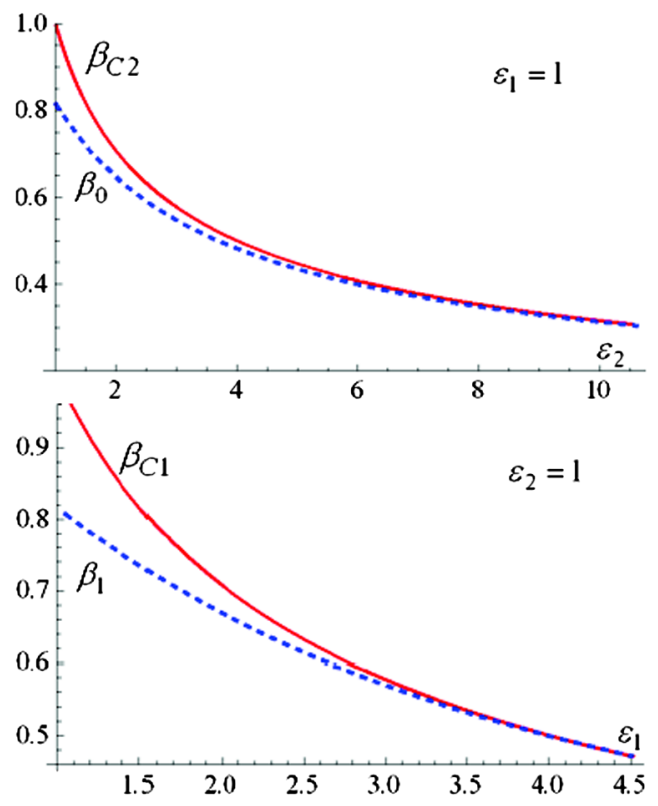

FIG. 4. The dependences of the threshold velocities $\beta_{C 2}, \beta_{C 1}$ (solid red line) and $\beta_{0}, \beta_{1}$ (dashed blue line) on the permittivity $\varepsilon_{2}$ or $\varepsilon_{1}$, respectively; the KEG is positive for $\beta<\beta_{0}$ if $\varepsilon_{1}=1$ (top), and for $\beta_{1}<\beta<\beta_{C 1}$ if $\varepsilon_{2}=1$ (bottom). than for velocities closed to $\beta_{C 2}$ [Fig. 6(c)]. This results in a principal difference in the integral characteristic behavior (17): if $t \rightarrow \infty$ then $\Delta A_{n}$ tends to a positive value when $\beta<\beta_{0}$ [Figs. 6(d) and 6(e)] and to a negative one when $\beta>\beta_{0}$ [Fig. 6(f)].

The case of motion of the charge from dielectric into vacuum is presented in Fig. 7. The KEG per unit length is always negative for $t<0$. After flying of the particle into vacuum, $d A_{n} / d z$ remains negative during some time
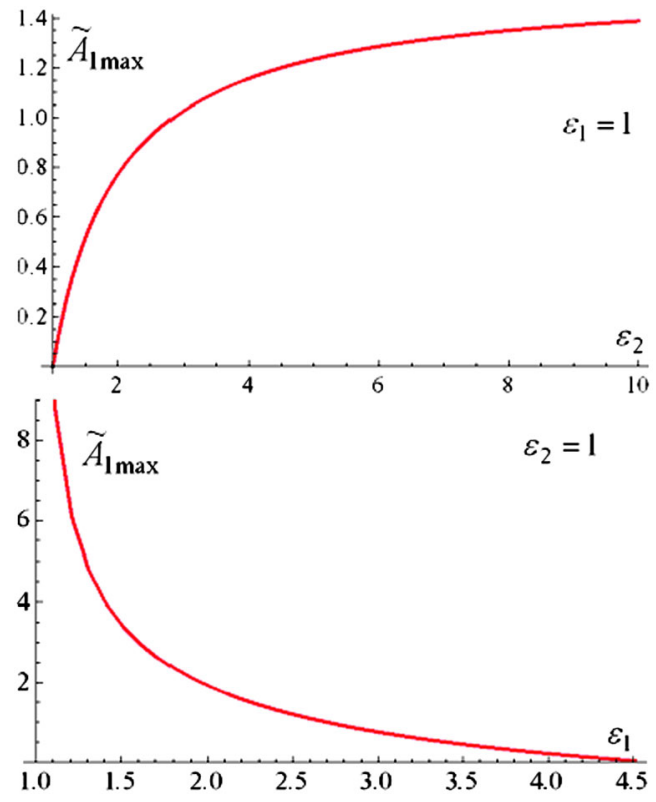

FIG. 5. The dependence of the maximum value of the KEG on the permittivity $\varepsilon_{2}$ or $\varepsilon_{1}$ : for $\varepsilon_{1}=1$ at $\beta \rightarrow 0$ (top), and for $\varepsilon_{2}=1$ at $\beta \rightarrow \beta_{C 1}$ (bottom). 


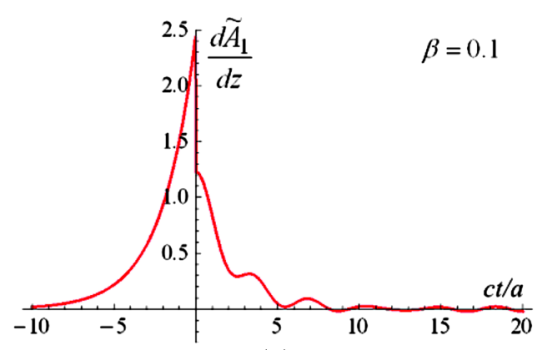

(a)

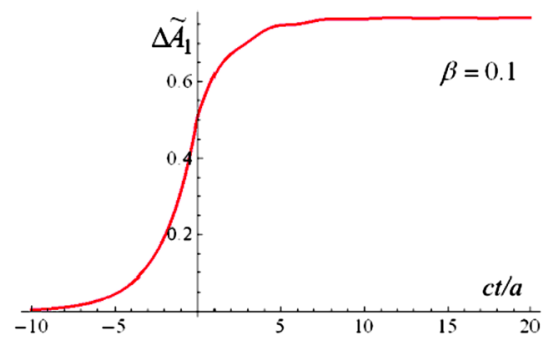

(d)

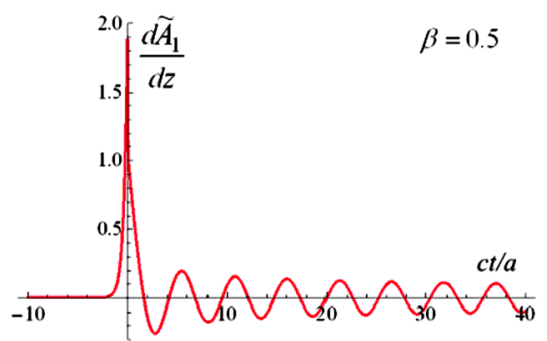

(b)

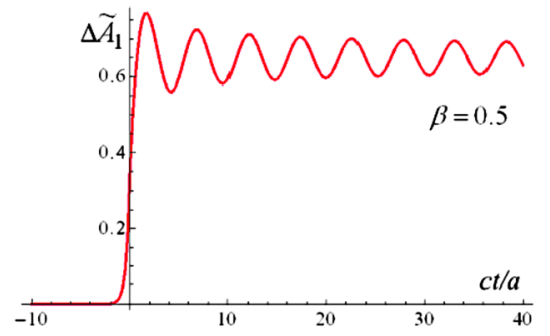

(e)

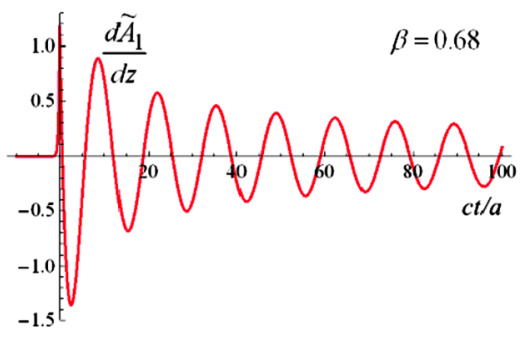

(c)

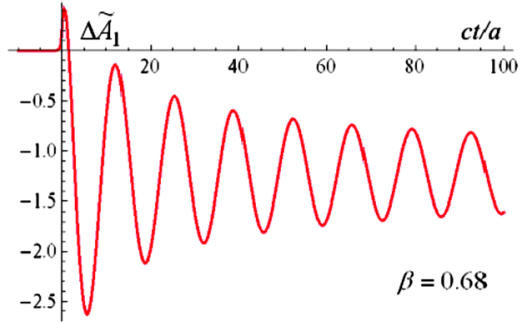

(f)

FIG. 6. The case of flying from vacuum into dielectric: the time dependences of the normalized field work per unit length $d \tilde{A}_{1} / d z=$ $a^{2} q^{-2} d A_{1} / d z$ (top) and the normalized field work $\Delta \tilde{A}_{1}(t)=a q^{-2} \Delta A_{1}(t)$ (bottom) of the 1st mode for different values of $\beta$; $\varepsilon_{1}=1$, $\varepsilon_{2}=2, \beta_{C 2}=0.707$, and $\beta_{0}=0.646$; values of $\beta$ are shown in the figures.

interval, and then oscillates near the zero level because of the interaction between the charge and transition radiation [Figs. 7(a)-7(c)]. The time interval with negative magnitude of $d A_{n} / d z$ is getting negligibly small with increase in $\beta$. The amplitude of oscillations at $t>0$ increases with increase in $\beta$. The area of positive values of $d A_{n} / d z$ is getting more significant for velocities closed to $\beta_{C 1}$ [Fig. 7(c)]. The integral characteristic $\Delta A_{n}$ tends (at $t \rightarrow \infty)$ to some negative value for $\beta<\beta_{1}$ [Figs. 7(d) and 7(e)] and to positive one for $\beta_{1}<\beta<\beta_{C 1}$ [Fig. 7(f)].

\section{CONCLUDING REMARKS}

We consider the KEG for the separate mode. The total KEG is the sum of the mode contributions: $A=\sum_{n} A_{n}$. However, there are some restrictions on the number of modes that are included in the sum. These restrictions are related to factors such as the bunch size, the vacuum channel radius, etc. Specifically, our assumption on the inessentiality of the vacuum channel holds true if the channel radius $b$ is less than the mode field variation scale $\sim a / n$. Essentially, the modes with numbers $n \gg a / b$ do

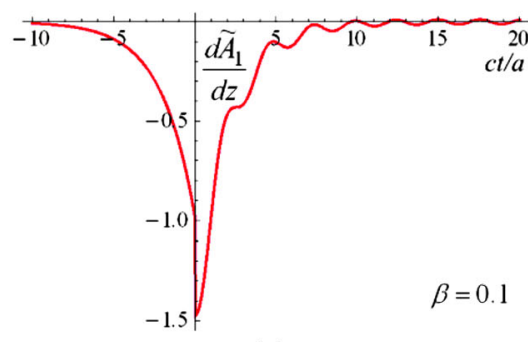

(a)

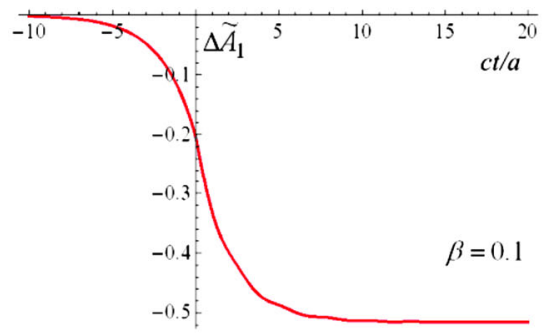

(d)

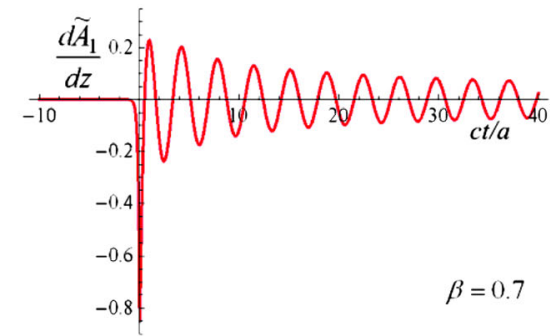

(b)

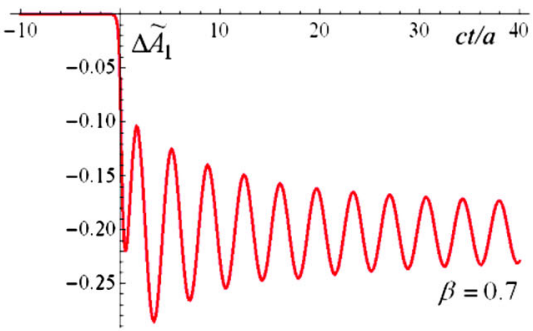

(e)

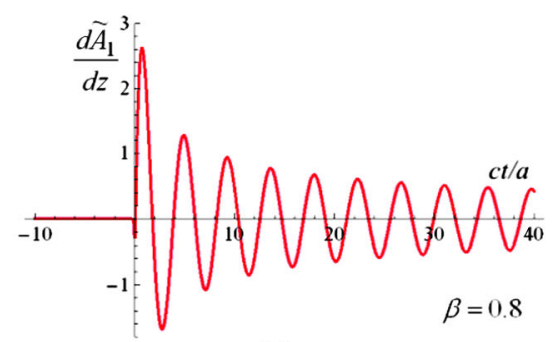

(c)

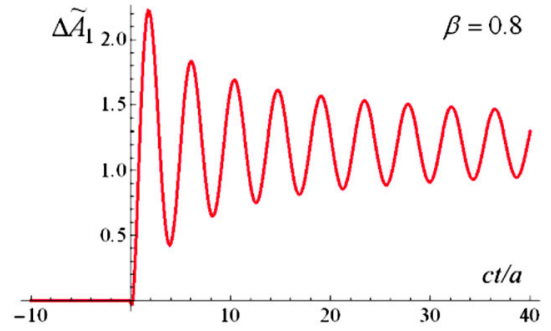

(f)

FIG. 7. The case of flying from dielectric into vacuum: the same as in Fig. 6 for parameters $\varepsilon_{1}=1.5, \varepsilon_{2}=1, \beta_{C 1}=0.816$, and $\beta_{1}=0.735$. 
not interact with the dielectric and do not contribute to the KEG. An analogous restriction is imposed due to the finite size of the bunch $\sigma$. Thus the number of modes contributed in the KEG can be estimated as $n_{\max } \sim \min \{a / b, a / \sigma\}$.

An upper estimate of the KEG of electrons or protons can be obtained using approximations (13) and (15). For a bunch with $N$ particles that have charge $e$, the estimate of the maximum value of the KEG per particle ( $\delta K_{n}=A_{n} / N$ ) for the separate mode is

$$
\begin{gathered}
\delta K_{n} \leq \delta K_{n}^{(0)} \frac{\left(\varepsilon_{2}-1\right)}{\varepsilon_{2}} \quad \text { for } \varepsilon_{1}=1, \\
\delta K_{n} \leq \delta K_{n}^{(0)} \frac{\left[1+\varepsilon_{1}^{2}-\left(\varepsilon_{1}-1\right) \sqrt{\varepsilon_{1}\left(2 \varepsilon_{1}-1\right)}\right]}{\sqrt{\varepsilon_{1}\left(\varepsilon_{1}-1\right)}\left[1-\varepsilon_{1}+\sqrt{\varepsilon_{1}\left(2 \varepsilon_{1}-1\right)}\right]} \text { for } \varepsilon_{2}=1,
\end{gathered}
$$

where

$$
\delta K_{n}^{(0)}=\frac{e^{2} N}{a \chi_{n} J_{1}^{2}\left(\chi_{n}\right)} \approx \frac{1.44 \times 10^{-7} \cdot N}{a \chi_{n} J_{1}^{2}\left(\chi_{n}\right)}(\mathrm{eV}) .
$$

For example, if $a \sim 1 \mathrm{~cm}$ then we obtain $\delta K_{n}^{(0)} \sim$ $1 \mathrm{KeV}$ for a nano-Coulomb bunch $\left(N \sim 10^{10}\right)$ and $\delta K_{n}^{(0)} \sim 100 \mathrm{KeV}$ for a bunch with $q \sim 100 \mathrm{nC}\left(N \sim 10^{12}\right)$. Note that bunches with charge $\sim 1-100 \mathrm{nC}$ are actively used in modern dielectric accelerating structures $[9,10]$. The KEG estimations obtained above are not large, but the total KEG can be essentially larger for a number of reasons.

First, it should be emphasized that, in the case $\varepsilon_{2}=1$, $\delta K_{n}$ can be much more than $\delta K_{n}^{(0)}$ if $\varepsilon_{1}-1 \ll 1$. In this case, in accordance with (19) we have

$$
\delta K_{n} \leq \delta K_{n}^{(0)} \frac{2}{\sqrt{\varepsilon_{1}-1}} .
$$

Therefore essential acceleration of ultrarelativistic particles can be achieved if the bunch flies out the dielectric with small enough permittivity. For example, if $\varepsilon_{1}=1.01$ then $\delta K_{n} \leq 20 \delta K_{n}^{(0)}$, that is $\delta K_{n} \leq 20 \mathrm{KeV}$ for $q \sim 1 \mathrm{nC}$ and $\delta K_{n} \leq 2 \mathrm{MeV}$ for $q \sim 100 \mathrm{nC}$ (as earlier we consider $a \sim 1 \mathrm{~cm})$.

Second, the KEG can be increased by using waveguides with smaller radius (some modern structures have the waveguide radius $\sim 2 \mathrm{~mm} \mathrm{[10]).}$

Third, we have to take into account that the estimations above concern one mode only. The total KEG $\delta K_{\Sigma}$ can be much more than $\delta K_{n}$ because of the coherent summation of contributions of different modes. Note that $\delta K_{n}$ weakly depends on the mode number $n$. Therefore $\delta K_{\Sigma} \sim$ $n_{\max } \delta K_{1}$ where $n_{\max }$ is a number of modes with wavelengths which are more than the bunch length. Taking into account that the bunches with $q \sim 1-100 \mathrm{nC}$ can have the length $\sim 2 \mathrm{~mm}$ [9], one can obtain that $n_{\max }$ can be $\sim 2-5$.

In our opinion, the factors indicated above can result in the total KEG $\sim 100 \mathrm{KeV}$ for the nano-Coulomb bunch and $\sim 10 \mathrm{MeV}$ for the bunch having the charge $100 \mathrm{nC}$. Such an increase of particle energy can be interesting, for example, for medical and industrial accelerators.

Note that estimations made above concern relatively large bunches. For microbunches the KEG decreases because of small value of the charge $(q \sim 100 \mathrm{pC})$. However, in this case, waveguides with radii $\sim 100 \mu \mathrm{m}$ can be applied [11]. Therefore the total KEG $\sim 1 \mathrm{MeV}$ looks realistic for such bunches as well.

\section{ACKNOWLEDGMENTS}

This research was supported by Saint Petersburg State University.

[1] S. A. Kheifets and S. A. Heifets, Report No. SLAC-PUB3965, 1986.

[2] L. Palumbo, Part. Accel. 25, 201 (1990).

[3] L. Palumbo, V. G. Vaccaro, and M. Zobov, Wake Fields and Impedance (CAS Advanced School on Accelerator Physics, Rhodes, Greece, 1994).

[4] V.L. Ginzburg and V.N. Tsytovich, Transition Radiation and Transition Scattering (Taylor \& Francis, London, 1990).

[5] B. M. Bolotovskii, Sov. Phys. Usp. 4, 781 (1962).

[6] T. Yu. Alekhina and A. V. Tyukhtin, Phys. Rev. E 83, 066401 (2011).

[7] T. Yu. Alekhina and A. V. Tyukhtin, Phys. Rev. ST Accel. Beams 15, 091302 (2012).

[8] A.P. Prudnikov, Yu. A. Brychkov, and O.I. Marichev, Integrals and Series. Special Functions (Nauka, Moscow, 1983).

[9] W. Gai, AIP Conf. Proc. 1086, 3 (2009).

[10] A. Kanareykin, J. Phys. Conf. Ser. 236, 012032 (2010).

[11] A. M. Cook, R. Tikhoplav, S. Y. Tochitsky, G. Travish, O.B. Williams, and J.B. Rosenzweig, Phys. Rev. Lett. 103, 095003 (2009). 